\title{
Fabrication of Hybrid Organic Photovoltaic Devices Using Electrostatic Spray Method
}

\author{
Zhe-Wei Chiu, ${ }^{1}$ Yu-Jen Hsiao, ${ }^{2}$ Te-Hua Fang, ${ }^{1}$ and Liang-Wen $\mathrm{Ji}^{3}$ \\ ${ }^{1}$ Department of Mechanical Engineering, National Kaohsiung University of Applied Sciences, Kaohsiung 807, Taiwan \\ ${ }^{2}$ National Nano Device Laboratories, National Applied Research Laboratories, Tainan 741, Taiwan \\ ${ }^{3}$ Institute of Electro-Optical and Materials Science, National Formosa University, Yunlin 632, Taiwan
}

Correspondence should be addressed to Te-Hua Fang; fang.tehua@msa.hinet.net

Received 29 April 2014; Revised 24 June 2014; Accepted 2 July 2014; Published 4 August 2014

Academic Editor: Ting-Jen Hsueh

Copyright (C) 2014 Zhe-Wei Chiu et al. This is an open access article distributed under the Creative Commons Attribution License, which permits unrestricted use, distribution, and reproduction in any medium, provided the original work is properly cited.

\begin{abstract}
Hybrid organic photovoltaic devices (OPVDs) are fabricated using the electrostatic spray (e-spray) method and their optical and electrical properties are investigated. E-spray is used to deposit a hybrid film (P3HT: PCBM/nanodiamond) with morphology and optical characteristics onto OPVDs. The root-mean-square roughness and optical absorption increase with increasing nanodiamond content. The performance of e-spray is comparable to that of the spin-coating method under uniform conditions. The device takes advantage of the high current density, power conversion efficiency, and low cost. Nanodiamond improves the shortcircuit current density and power conversion efficiency. The best performance was obtained with $1.5 \mathrm{wt} \%$ nanodiamond content, with a current density of $7.28 \mathrm{~mA} / \mathrm{cm}^{2}$ and a power conversion efficiency of $2.25 \%$.
\end{abstract}

\section{Introduction}

Organic photovoltaic devices (OPVDs) have received attention for large-area manufacturing. The roll-to-roll printing process can be used to manufacture devices on flexible substrates with transparent electrode [1]. OPVDs can be fabricated using a solution-processed method, such as spincoating (SC), gravure printing, dip-coating, screen printing, inkjet printing, and the electrostatic spray (e-spray) method. OPVDs with an efficiency of as high as $8.3 \%$ have been demonstrated [2].

The e-spray technique has been applied to OPVDs due to their high surface to volume ratio. Polymers [3], biomaterials [4], and metal oxides [5] with small feature sizes have been fabricated using this method. The morphology of e-spray droplets must be carefully controlled via several parameters, such as applied voltage, distance between nozzle and substrate, pressure, and solution injection rate. The performance of OPVDs is determined by the spray diameter and evaporation speed [6].

A solar cloth of P3TH:PCBM blend was fabricated using the electrospinning technique [7]. The power conversion efficiency was up to $1.9 \%$ when the surface roughness was reduced by depositing a solvent consisting of odichlorobenzene and acetone via the e-spray method [6]. OPVDs with a power conversion efficiency that exceeds $3.25 \%$ have been produced using the e-spray method, which rearranges the boundaries and constructs more continuous interpenetrating networks by solvent vapor soaking treatment [8]. The e-spray method is a promising technique for the deposition of a polymer blend layer onto OPVDs with low cost, roll-to-roll processing, and large-area manufacturing. Nanodiamonds have received a lot of interest as a semiconductor material, due to their strong ultraviolet absorption and high thermal conductivity. Nanodiamonds (1.5 wt\%) embedded in OPVDs via the spin-coating method can enhance efficiency and reliability [9]. The active layer of OPVDs doped with nanodiamond with a grain size of $50 \mathrm{~nm}$ was shown to have the highest efficiency [10].

The present study investigates $\mathrm{P} 3 \mathrm{HT}$ :PCBM/nanodiamond hybrid film prepared for OPVDs using the e-spray method. The effects of nanodiamond content in OPVDs on the morphology, optical absorption, photoluminescence, and photovoltaic performance are determined. 


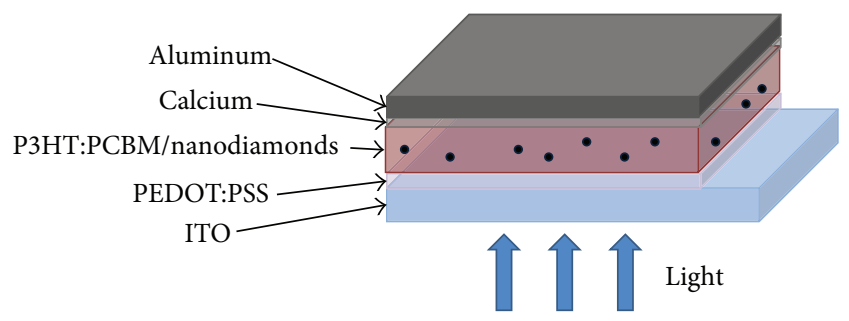

(a)

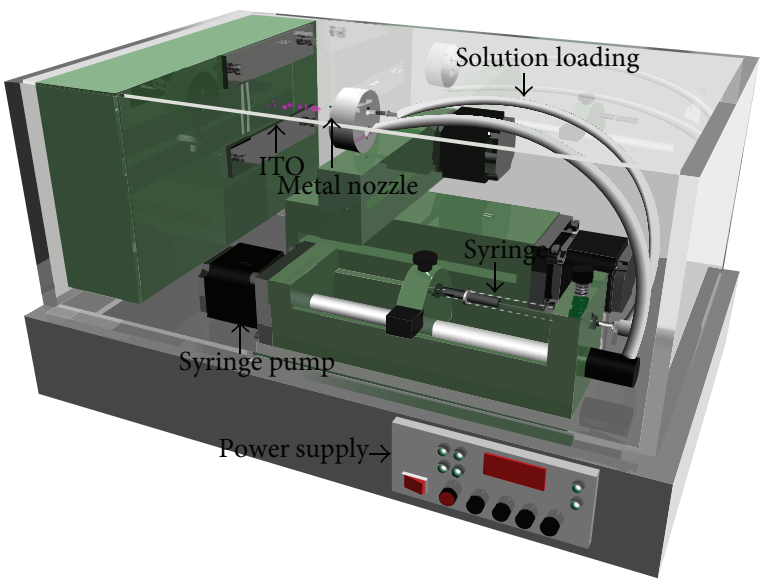

(b)

FIGURE 1: Schematics of (a) device structure with P3HT:PCBM/nanodiamond as the active layer and (b) e-spray method.

\section{Experimental Procedure}

Indium tin oxide (ITO) substrates were sequentially cleaned with acetone, isopropanol, and deionized water via ultrasonication for $10 \mathrm{~min}$ to remove contaminants and then etched with a pattern before being placed into a reactive ion etching system for oxygen plasma treatment to increase the ITO surface hydrophilicity. After PEDOT:PSS was spin-coated onto the substrates at $2600 \mathrm{rpm}$ for $1 \mathrm{~min}$, the samples were annealed at $120^{\circ} \mathrm{C}$ for $30 \mathrm{~min}$. The active layer consisted of P3HT (FEM Inc.) and PCBM (Nano-C) (at a weight ratio of $1: 0.8$ ) dissolved in 1,2-dichlorobenzene (DCB) with various concentrations of nanodiamond (average grain size of about $50 \mathrm{~nm}$ ). We have used nanodiamond (ND, ITRI Taiwan) of the sizes of about $50 \mathrm{~nm}$, prepared by the detonation method. Figure 1(a) shows the device structure of the hybrid OPVDs in which the P3HT:PCBM/nanodiamond film was the active layer. A schematic of the e-spray method is shown in Figure 1(b). The distance between the metal nozzle and the substrate was $12 \mathrm{~cm}$, the spray area was $6 \mathrm{~cm}^{2}$, and the applied voltage was $10 \mathrm{kV}$. The hybrid solution was injected at a rate of $10 \mu \mathrm{L} \cdot \mathrm{min}^{-1}$. The e-spray was applied to the substrate 12 times, each for 20 seconds. The active layer was annealed at $120^{\circ} \mathrm{C}$ for 15 min after being allowed to dry naturally. Then, a Ca buffer layer $(25 \mathrm{~nm})$ and an $\mathrm{Al}$ electrode $(100 \mathrm{~nm})$ were deposited onto the substrate via thermal evaporation. The active area was about $0.04 \mathrm{~cm}^{2}$. All deposition processes were conducted at room temperature.

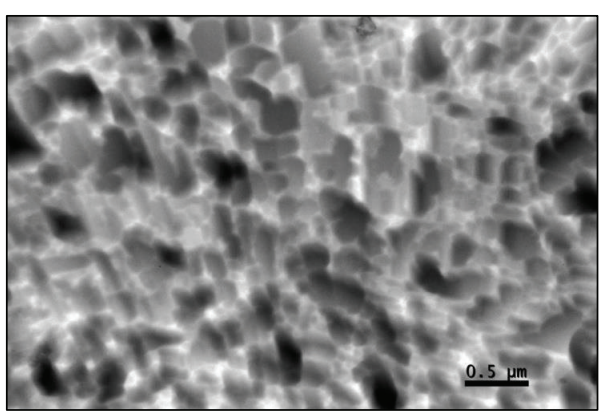

(a)

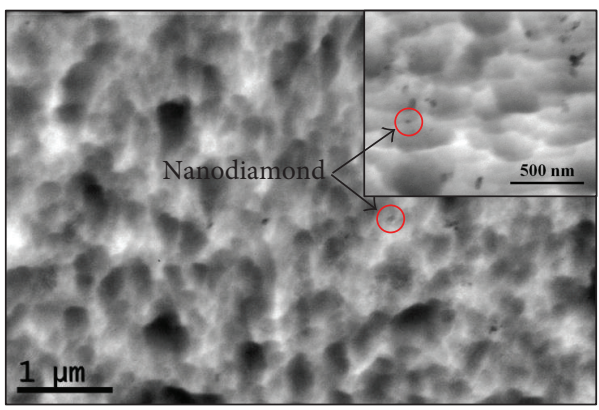

(b)

FIGURE 2: TEM images of films deposited by e-spray method: (a) P3HT:PCBM and (b) P3HT:PCBM doped with $1.5 \mathrm{wt} \%$ nanodiamond.

The morphology of the photoactive layer was measured with transmission electron microscopy (TEM) and atomic force microscopy (AFM). The absorption spectra were obtained using an ultraviolet-visible (UV-Vis) spectrometer (Hitachi, U-3010). The current-voltage measurements were obtained using a source meter (Keithley, 2410) and a solar simulator (TELTEC) with an AM1.5 filter under an irradiation intensity of $100 \mathrm{mV} \mathrm{cm}^{-2}$. For external quantum efficiency (EQE) measurements, a xenon lamp (Oriel, 66150, $75 \mathrm{~W}$ ) was used as the light source. All the samples were measured at room temperature.

\section{Results and Discussion}

The active layer was deposited via the e-spray method in a glove box and then annealed to reduce contact resistance with the electrodes. The crystallinity and hole transport of $\mathrm{P} 3 \mathrm{HT}$ increased after annealing at $120^{\circ} \mathrm{C}$ [8]. By electrostatic spraying of the mixed liquid, the solution will be atomization form mutually exclusive electronic, the uniform surface on the substrate form atomized mixture. Spin-coating was used to form a smooth film surface. The surfaces of the films after e-spray deposition were quite rough, which is attributed to the evaporation of the solution and the solution injection rate. The morphology of the droplets was affected by the electron transfer of OPVDs. E-spray deposition must be determined before the film is already naturally dried because the performance of OPVDs would seriously be affected by morphology of the film surface. The parameters of the e-spray process must be carefully controlled. E-spray deposition uses 


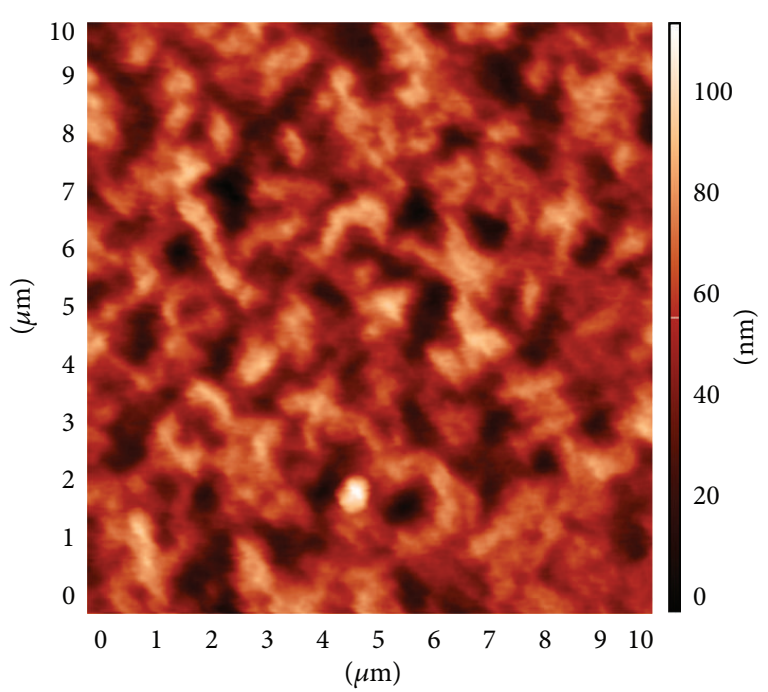

(a)

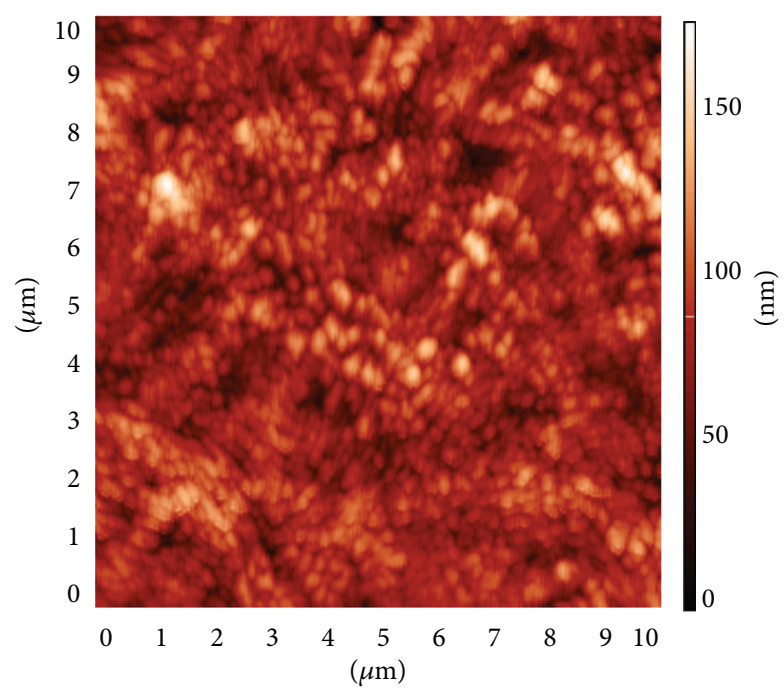

(c)

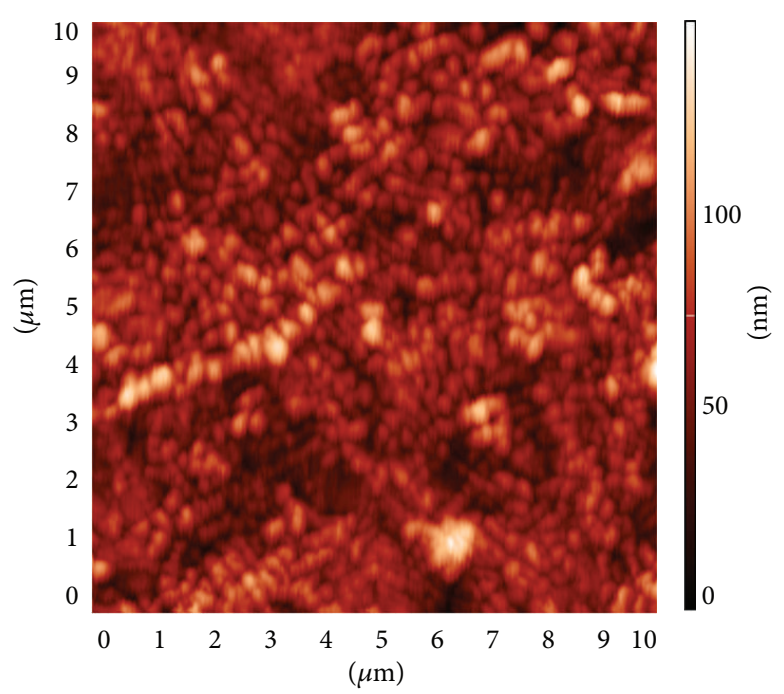

(b)

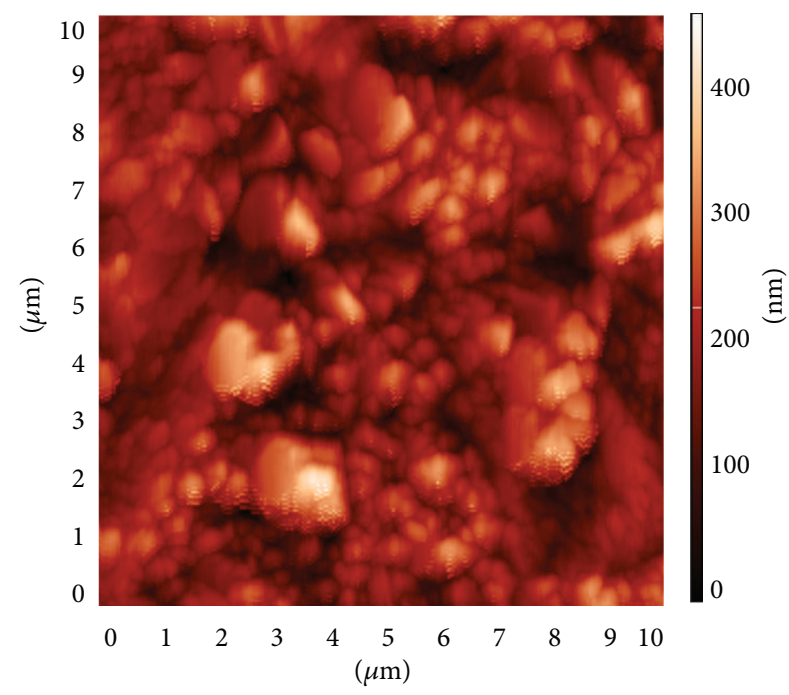

(d)

FIGURE 3: AFM images of P3HT:PCBM film doped with nanodiamond at concentrations of (a) 0, (b) 0.5, (c) 1.5, and (d) 3 wt\%.

$10 \%$ less polymer material than does the spin-coating method for a given film thickness. Using the e-spray method for the fabrication of the active layer of OPVDs thus reduces costs. The nanodiamond was dispersed in different film. The metal electrode has bad contact cause recombination of electrons and holes, this results induce e-spray has obvious irregular structure.

TEM images of PEDOT:PSS/P3HT:PCBM/nanodiamond film prepared via the e-spray method are shown in Figure 2. The grain size of P3HT:PCBM film (see Figure 1(a)) is about $200 \mathrm{~nm}$. Particles aggregated to form large agglomerates, which then form the composite film with a continuous network structure. The surface area, number of donor/acceptor interfaces of the active layer, and thus charge generation were increased by the e-spray method. Figure 2(b) shows a TEM image of P3HT:PCBM/nanodiamond film. The nanodiamond was dispersed well and embedded in the P3HT:PCBM (1:0.8) polymer quite uniformly. The average diameter of the nanodiamond was about $50 \mathrm{~nm}$. OPVDS with this nanodiamond size exhibited highest power conversion efficiency in a previous study [10].

Figures 3(a)-3(d) show AFM images of P3HT:PCBM doped with nanodiamond at concentrations of $0,0.5,1.5$, and $3 \mathrm{wt} \%$, respectively. The e-sprayed films had root-meansquare (RMS) roughness values of 16.1, 17.3, 19.1, and $56.6 \mathrm{~nm}$, respectively. The spin-coated film had an RMS roughness of $2.6 \mathrm{~nm}$. The RMS roughness is higher for the e-sprayed films due to the stack deposition of spray droplets. The AFM surface morphology is shown high concentrations of nanodiamond induce the highest rougher surface.

Figure 4 shows optical absorption spectra for P3HT: PCBM doped with nanodiamond at concentrations of $0,0.5$, 1.5 , and $3 \mathrm{wt} \%$, respectively. The absorption in the range of 400 to $650 \mathrm{~nm}$ is attributed to the absorption of P3HT [11]. 


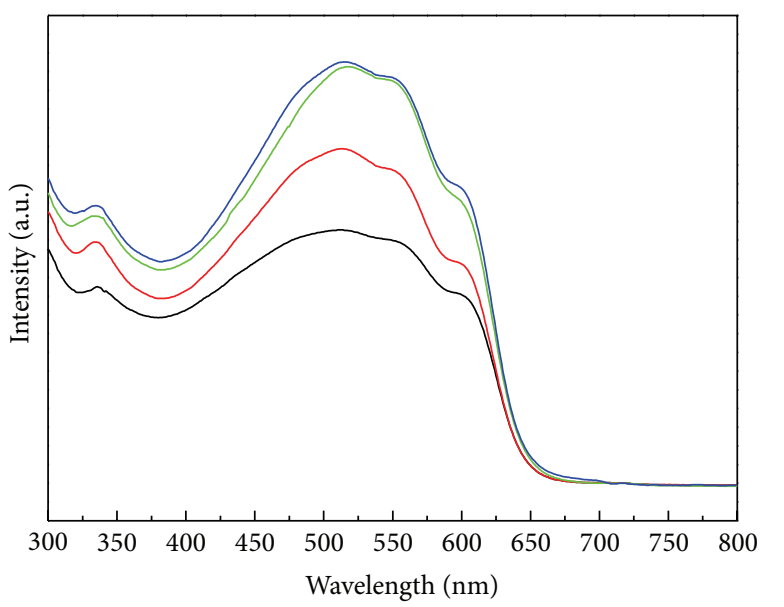

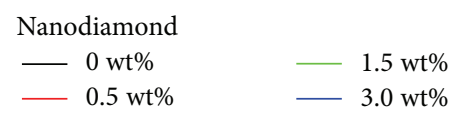

FIGURE 4: Absorption spectra of hybrid P3HT:PCBM film doped with nanodiamond at various concentrations.

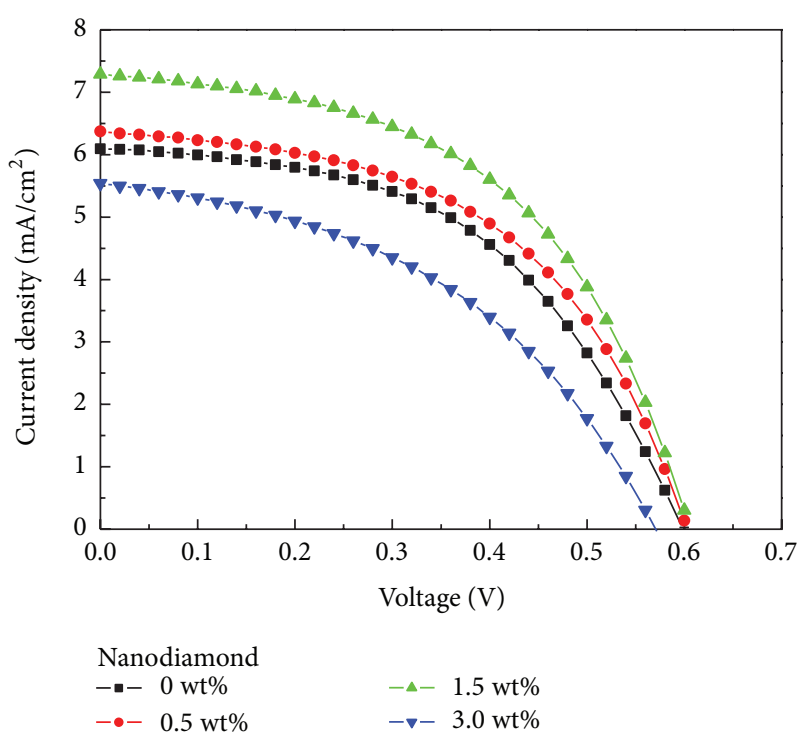

FIGURE 5: Current density-voltage characteristics of hybrid OPVDs doped with nanodiamond at various concentrations, under AM1.5G at $100 \mathrm{~mW} \cdot \mathrm{cm}^{-2}$ illumination.

The spectrum for the active film exhibited a small red shift when the nanodiamond concentration was increased from 0 to $3 \mathrm{wt} \%$. The absorption intensity increased with increasing nanodiamond concentration. The nanodiamond created a scattering effect, which can lead to better absorption via the active layer. The doping nanodiamond in organic solar cell can increase the scattering effect of sunlight effectively. It could be attributed to the reflection and refraction of sunlight in organic hybrid active layer. The absorption intensity thus increased with increasing generation of electron-hole pairs by the nanodiamond, which enhances power conversion efficiency [12].
TABLE 1: Photovoltaic performance of investigated OPVDs doped with nanodiamond at various concentrations measured under AM1.5G at $100 \mathrm{~mW} \cdot \mathrm{cm}^{-2}$ illumination.

\begin{tabular}{lcccc}
\hline & $\begin{array}{c}V_{\text {oc }} \\
(\mathrm{V})\end{array}$ & $\begin{array}{c}J_{\mathrm{sc}} \\
\left(\mathrm{mA} / \mathrm{cm}^{2}\right)\end{array}$ & $\begin{array}{c}\mathrm{FF} \\
(\%)\end{array}$ & $\begin{array}{c}\eta \\
(\%)\end{array}$ \\
\hline Standard & 0.60 & 6.10 & 49.87 & 1.82 \\
$0.5 \%$ & 0.60 & 6.37 & 51.33 & 1.96 \\
$1.5 \%$ & 0.60 & 7.28 & 51.42 & 2.25 \\
$3.0 \%$ & 0.58 & 5.54 & 43.04 & 1.38 \\
\hline
\end{tabular}

The performance of OPVDs with active film doped with nanodiamond at various concentrations is shown in Table 1. Figure 5 shows the current density-voltage $(J-V)$ characteristics of the e-sprayed hybrid OPVDs measured under an illumination intensity of $100 \mathrm{~mW} \cdot \mathrm{cm}^{-2}$. The photovoltaic performance of the $400 \mathrm{~nm}$ thick active layer spincoated at $600 \mathrm{rpm}$ was very similar to that of the e-sprayed layer. The open-circuit voltage, short-circuit current density, fill factor, and power conversion efficiency values of the OPVD obtained using the spin-coating method are $0.6 \mathrm{~V}$, $5.4 \mathrm{~mA} \cdot \mathrm{cm}^{-2}, 56.27 \%$, and $1.82 \%$, respectively. The shortcircuit current density for the e-sprayed active layer is higher than that of the spin-coated layer. The fill factor of the esprayed active layer is lower because the active layer/metal electrode has imperfect contact with the roughness surface.

Hybrid OPVDs with P3HT:PCBM/nanodiamond films with nanodiamond levels of $0,0.5,1.5$, and $3 \mathrm{wt} \%$ have power conversion efficiency values of $1.82 \%, 1.96 \%, 2.25 \%$, and $1.38 \%$, respectively. Nanodiamond improves the current density for concentrations up to $1.5 \mathrm{wt} \%$, which is attributed to the absorption increase by the e-spray active layer. The results reveal that $1.5 \mathrm{wt} \%$ nanodiamond doping into OPVDs increases the short-circuit current density from 6.1 to $7.28 \mathrm{~mA} \cdot \mathrm{cm}^{-2}$. The power conversion efficiency of the hybrid OPVDs improved from $1.82 \%$ to $2.25 \%$. However, when the concentration of nanodiamond exceeds $3 \mathrm{wt} \%$, the efficiency decreases due to an increase in leakage current $[13,14]$. In this study, we have achieved the performance $2.25 \pm 0.1 \%$. The difference may lie in preparation of OPV hybrid film (e.g., process condition, machine, and material differences).

Figure 6 shows the EQE spectra for hybrid OPVDs with various nanodiamond concentrations measured at room temperature. The EQE spectra of the P3HT:PCBM/nanodiamond show a high conversion efficiency in the range of 300 to $650 \mathrm{~nm}$ [15]. Although all EQE spectra are similar in shape, the EQE values for the OPVDs doped with $1.5 \mathrm{wt} \%$ nanodiamond are higher than those for OPVDs doped with $0,0.5$, and $3 \mathrm{wt} \%$ nanodiamond for all wavelengths. This difference is due to an increase in the rate of exciton generation and increased probability of exciton dissociation, which enhance the short-circuit current density for P3HT:PCBM doped with $1.5 \mathrm{wt} \%$ nanodiamond.

\section{Conclusion}

E-sprayed P3HT:PCBM films doped with nanodiamond at various concentrations were investigated. Active layer has 


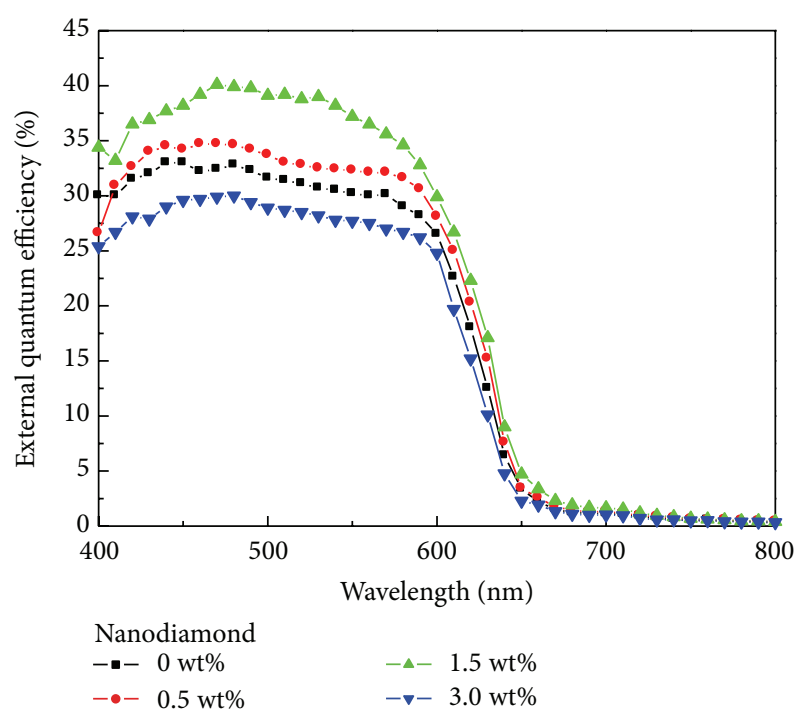

FIGURE 6: External quantum efficiency spectra of hybrid OPVDs doped with nanodiamond at various concentrations.

similarly stacked at e-spray method, AFM has the same trend when nanodiamond is over $1.5 \mathrm{wt} \%$, and root-mean-square (RMS) roughness has significantly increased. Absorption spectra of both the intensities increase with the concentration being increased. The results reveal that $1.5 \mathrm{wt} \%$ nanodiamond doping into OPVDs increases the short-circuit current density from 6.1 to $7.28 \mathrm{~mA} \cdot \mathrm{cm}^{-2}$ at e-spray method, which is due to e-spray increase interface with PEDOT:PSS and active layer. The e-spray is worse than spin-coating with fill factor, maybe the irregular structure form e-spray and metal electrode has bad contact cause recombination of electrons and holes. The best photovoltaic performance, in terms of short-circuit current density and power conversion efficiency, was obtained with $1.5 \mathrm{wt} \%$ nanodiamond doping. The espray method can possibly be used for flexible and large-area manufacturing.

\section{Conflict of Interests}

The authors declare that there is no conflict of interests regarding the publication of this paper.

\section{Acknowledgment}

The authors acknowledge the National Science Council of Taiwan for financially supporting this research under Grant NSC 100-2628-E-151-003-MY3.

\section{References}

[1] H. F. Dam and F. C. Krebs, "Simple roll coater with variable coating and temperature control for printed polymer solar cells," Solar Energy Materials and Solar Cells, vol. 97, pp. 191-196, 2012.
[2] B. Ratier, J. Nunzi, M. Aldissi, T. M. Kraft, and E. Buncel, "Organic solar cell materials and active layer designsimprovements with carbon nanotubes: a review," Polymer International, vol. 61, no. 3, pp. 342-354, 2012.

[3] Y. H. Lee, F. Mei, M. Y. Bai, S. Zhao, and D. R. Chen, "Release profile characteristics of biodegradable-polymer-coated drug particles fabricated by dual-capillary electrospray," Journal of Controlled Release, vol. 145, no. 1, pp. 58-65, 2010.

[4] S. Guha, M. Li, M. J. Tarlov, and M. R. Zachariah, "Electrospraydifferential mobility analysis of bionanoparticles," Trends in Biotechnology, vol. 30, no. 5, pp. 291-300, 2012.

[5] L. Wang, H. W. Xu, P. C. Chen, D. W. Zhang, C. X. Ding, and C. $\mathrm{H}$. Chen, "Electrostatic spray deposition of porous $\mathrm{Fe}_{2} \mathrm{O}_{3}$ thin films as anode material with improved electrochemical performance for lithium-ion batteries," Journal of Power Sources, vol. 193, no. 2, pp. 846-850, 2009.

[6] T. Fukuda, K. Takagi, T. Asano et al., "Bulk heterojunction organic photovoltaic cell fabricated by the electrospray deposition method using mixed organic solvent," Physica Status Solidi-Rapid Research Letters, vol. 5, no. 7, pp. 229-231, 2011.

[7] S. Sundarrajan, R. Murugan, A. S. Nair, and S. Ramakrishna, "Fabrication of P3HT/PCBM solar cloth by electrospinning technique," Materials Letters, vol. 64, no. 21, pp. 2369-2372, 2010.

[8] J. Kim, W. Chung, K. Kim et al., "Performance optimization of polymer solar cells using electrostatically sprayed photoactive layers," Advanced Functional Materials, vol. 20, no. 20, pp. 35383546, 2010.

[9] Y. J. Hsiao, T. H. Fang, L. W. Ji, Y. C. Lee, and B. T. Dai, “Size effect of nanodiamonds on P3HT:PCBM heterojunction solar cells," Electrochemistry Communications, vol. 18, no. 1, pp. 4-7, 2012.

[10] Y. Hsiao, T. Fang, L. Ji, Y. Lee, and B. Dai, "Nanodiamonds embedded in P3HT:PCBM for enhancing efficiency and reliability of hybrid photovoltaics," Electrochemical and Solid-State Letters, vol. 15, no. 4, pp. K27-K30, 2012.

[11] F. Wang, Z. Chen, L. Xiao, B. Qu, and Q. Gong, "Enhancement of the power conversion efficiency by expanding the absorption spectrum with fluorescence layers," Optics Express, vol. 19, no. S3, pp. A361-A368, 2011.

[12] V. Kruefu, E. Peterson, C. Khantha, C. Siriwong, S. Phanichphant, and D. L. Carroll, "Flame-made niobium doped zinc oxide nanoparticles in bulk heterojunction solar cells," Applied Physics Letters, vol. 97, no. 5, Article ID 053302, 2010.

[13] Z. W. Chiu, Y. J. Hsiao, T. H. Fang, and L. W. Ji, "Morphology and optical performance of graphene-doped organic photovoltaic cells," Microsystem Technologies, vol. 19, pp. 1781-1785, 2013.

[14] S. H. Wang, Y. J. Hsiao, T. H. Fang, M. H. Lin, and S. H. Kang, "Enhancing performance and nanomechanical properties of carbon nanotube doped P3HT:PCBM solar cells," ECS Journal of Solid State Science and Technology, vol. 2, no. 11, pp. M52M55, 2013.

[15] E. Voroshazi, B. Verreet, T. Aernouts, and P. Heremans, "Longterm operational lifetime and degradation analysis of P3HT: PCBM photovoltaic cells," Solar Energy Materials and Solar Cells, vol. 95, no. 5, pp. 1303-1307, 2011. 

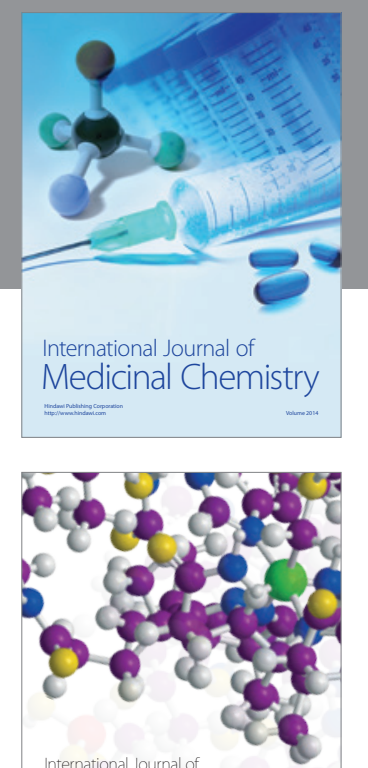

\section{Carbohydrate} Chemistry

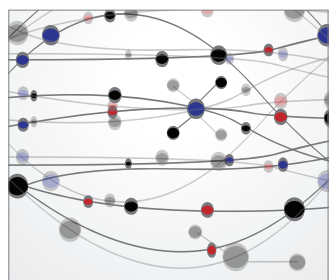

The Scientific World Journal
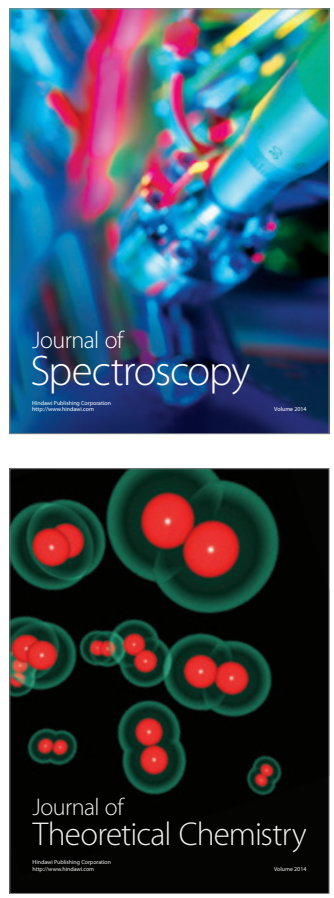
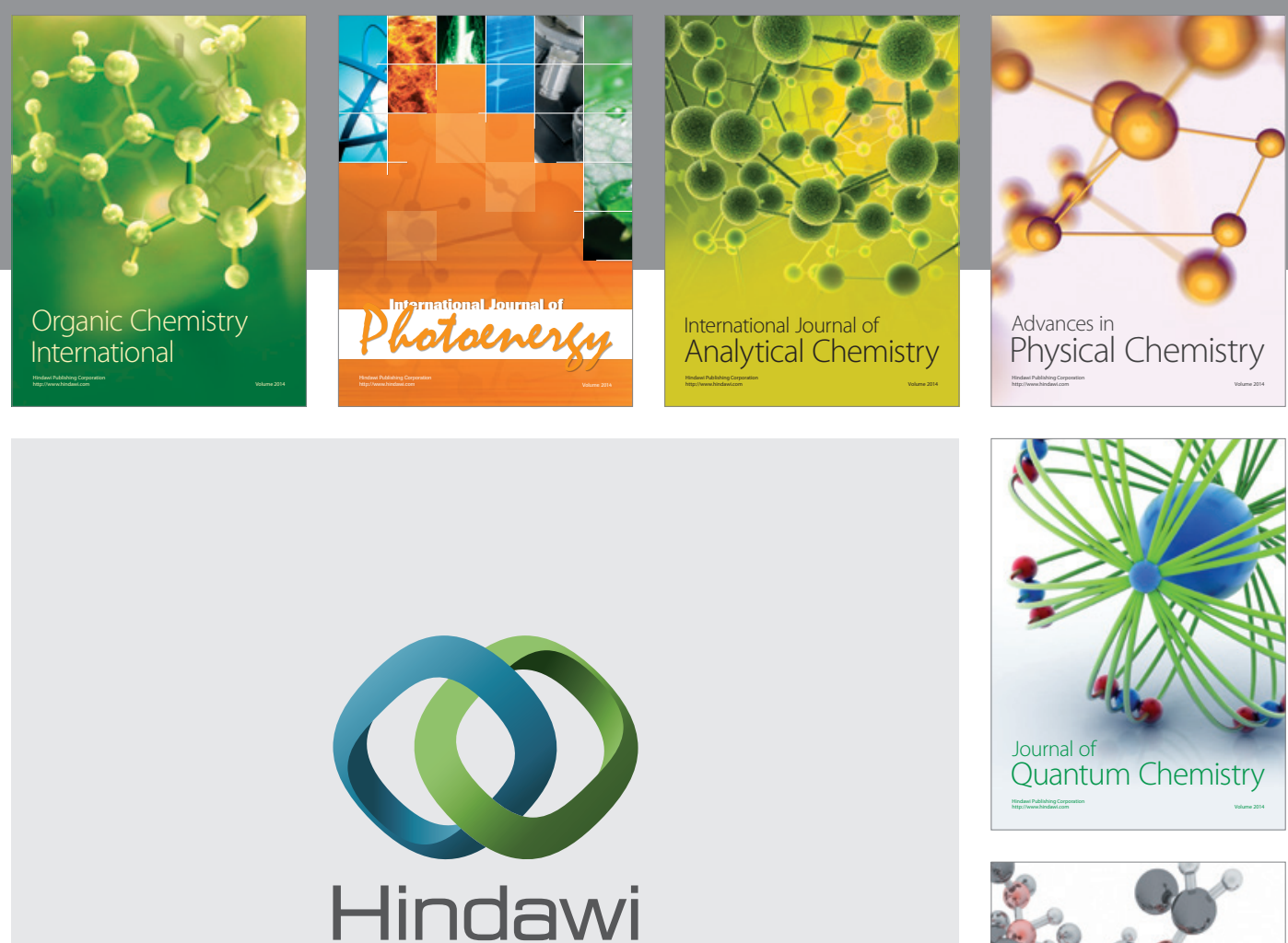

Submit your manuscripts at

http://www.hindawi.com

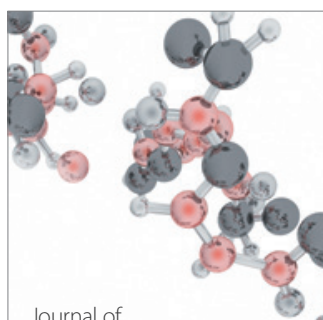

Analytical Methods

in Chemistry

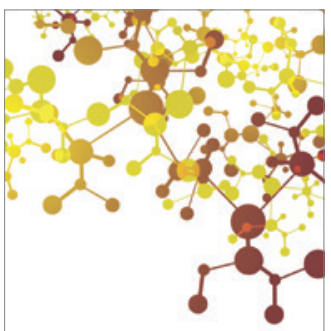

Journal of

Applied Chemistry

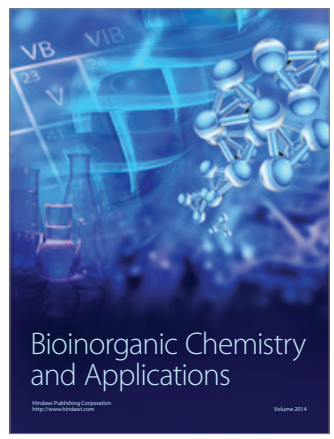

Inorganic Chemistry
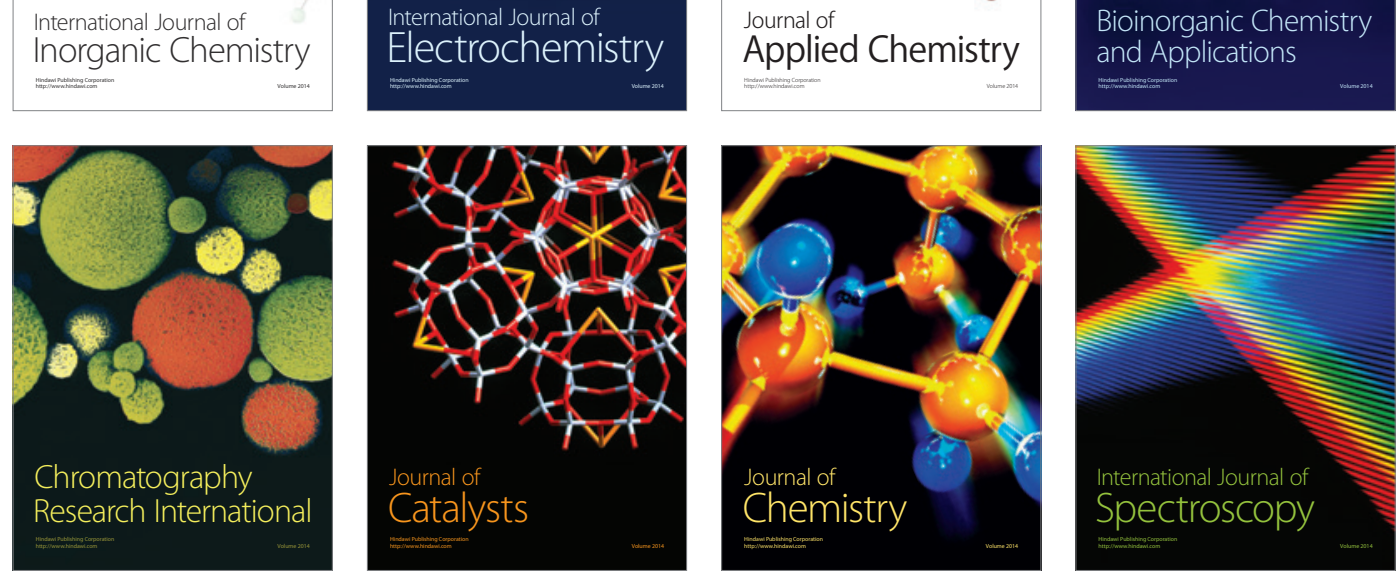